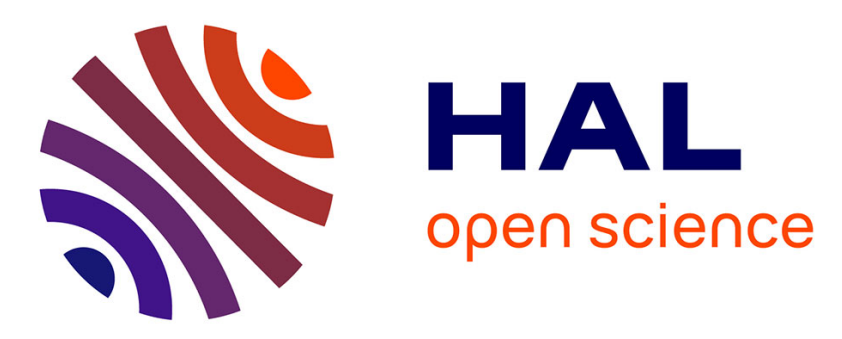

\title{
Convergent Strategy to Dizocilpine MK-801 and Derivatives
}

\author{
K. Harsha Vardhan Reddy, Expédite Yen-Pon, Sylvia Cohen-Kaminsky, Samir \\ Messaoudi, Mouad Alami
}

\section{- To cite this version:}

K. Harsha Vardhan Reddy, Expédite Yen-Pon, Sylvia Cohen-Kaminsky, Samir Messaoudi, Mouad Alami. Convergent Strategy to Dizocilpine MK-801 and Derivatives. Journal of Organic Chemistry, 2018, 83 (7), pp.4264-4269. 10.1021/acs.joc.8b00305 . hal-02411576

\section{HAL Id: hal-02411576 https://hal.science/hal-02411576}

Submitted on 15 Dec 2019

HAL is a multi-disciplinary open access archive for the deposit and dissemination of scientific research documents, whether they are published or not. The documents may come from teaching and research institutions in France or abroad, or from public or private research centers.
L'archive ouverte pluridisciplinaire HAL, est destinée au dépôt et à la diffusion de documents scientifiques de niveau recherche, publiés ou non, émanant des établissements d'enseignement et de recherche français ou étrangers, des laboratoires publics ou privés. 


\title{
Convergent Strategy to Dizocilpine MK-801 and Derivatives
}

\author{
K. Harsha Vardhan Reddy, ${ }^{\dagger, \#}$ Expédite Yen-Pon, ${ }^{\dagger, \#}$ Sylvia Cohen-Kaminsky, ${ }^{\ddagger} \S$ Samir Messaoudi, ${ }^{\dagger}{ }^{\dagger}$ (1) \\ and Mouad Alami* ${ }^{\dagger}$ \\ †Laboratoire de Chimie Thérapeutique, Faculté de Pharmacie, BioCIS, Univ. Paris-Sud, CNRS, University Paris-Saclay, \\ Châtenay-Malabry, F-92296, France \\ ${ }^{\ddagger}$ Inserm UMR_S 999, Hôpital Marie Lannelongue, Le Plessis-Robinson, F-92350, France \\ ${ }^{\S}$ Faculté de Médecine, Univ. Paris-Sud, Université Paris-Saclay, Le Kremlin-Bicêtre, F-94270, France
}

Supporting Information

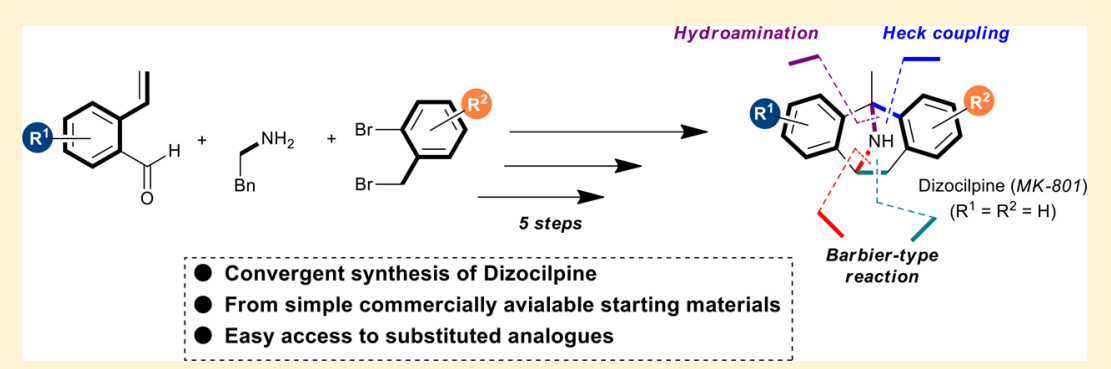

ABSTRACT: A convergent total synthesis of MK-801 has been achieved. Key synthetic transformations include a multicomponent Barbier-type reaction to construct the $\alpha$-branched amine, a selective Heck $\alpha$-coupling tactic to generate the exocyclic alkene skeleton, and a late-stage intramolecular hydroamination reaction between the exocyclic alkene and the secondary protected amine. The efficacy of this method was demonstrated by the synthesis of two news analogues substituted on the aromatic rings.

$M^{\prime}$ MK-801 is an original nortropane derivative whose core is fused with two benzene rings. One of its two isomers, the (+)-MK-801, ${ }^{1}$ also called dizocilpine, is a highly potent noncompetitive antagonist of the $N$-methyl-D-aspartate (NMDA) receptor, which acts by binding to a site located within the NMDA associated ion channel and thus prevents $\mathrm{Ca}^{2+}$ flux. This biological property gives it potential as a drug to treat CNS disorders, confirmed by its well-known use as an anticonvulsant. The therapeutic potency combined with the synthetic challenge triggered attention from several groups. The principal syntheses developed are summarized in Scheme 1. Shibasaki et al. ${ }^{2}$ reported the first enantioselective synthesis via a catalytic Reissert-type reaction starting from the 1methylisoquinoline using an aluminum-BINOL complex. Ivy Carroll and co-workers ${ }^{3}$ described a rapid synthesis of the racemic version of MK-801 based on a 1,3-dipolar cycloaddition between the betaine, generated in situ from the 4hydroxy-1-methylisoquinoline and a benzyne ring. The first partner could be prepared via a four-step route described by the same group. Molander et al. ${ }^{4}$ developed a synthetic pathway involving an original lanthanide-promoted intramolecular hydroamination reaction from the commercially available dibenzosuberone. The Merck lab ${ }^{5}$ approach consisted on the 1,2-addition of a Grignard on the dibenzosuberenone followed by a base-induced intramolecular cyclization and a final hydroxyl removal. Recently, Chen et al. $^{6}$ depicted a five-step synthetic route starting from the dibenzosuberenone with a key Lewis acid-catalyzed cyclization and a final magnesium desulfonylation. Although all these strategies are efficient, their main limitation is the introduction of substituents on both aromatic rings, which limits the pharmacomodulation of these parts of the molecules. Our group took up the challenge to develop a new pathway to synthesize this pharmaceutical agent and various analogues via a key multicomponent Barbier-type reaction that could tolerate a great diversity of modulations (Scheme 1).

The retrosynthetic approach we envisioned is depicted in Scheme 2. The nitrogen bridged core of dizocilpine could be obtained by an hydroamination reaction between the exocyclic alkene and the secondary protected amine of compound A, which could be obtained through an intramolecular selective $\alpha$ Heck reaction. The major milestone of our strategy was to obtain the preorganized vinyl/halide containing moiety $\mathbf{B}$ starting from the easily accessible 2 -vinylbenzaldehyde, ${ }^{7}$ the commercially available benzylamine, and 2-bromobenzyl bromide. Herein we report our preliminary results of this study.

Multicomponent reactions are very seducing for the synthesis of highly complex molecules because of the multiple bond formation usually achieved in one step. ${ }^{8} \mathrm{Wu}$ et al. ${ }^{9}$ and later Le Gall et al. ${ }^{10}$ developed an efficient zinc-promoted Barbier-like reaction that allows the access to $\alpha$-branched amines involving three partners: benzaldehyde, cyclic or alicyclic amine, and benzylbromide. As their methodology seems to tolerate a wide

Received: February 2, 2018

Published: February 28, 2018 
Scheme 1

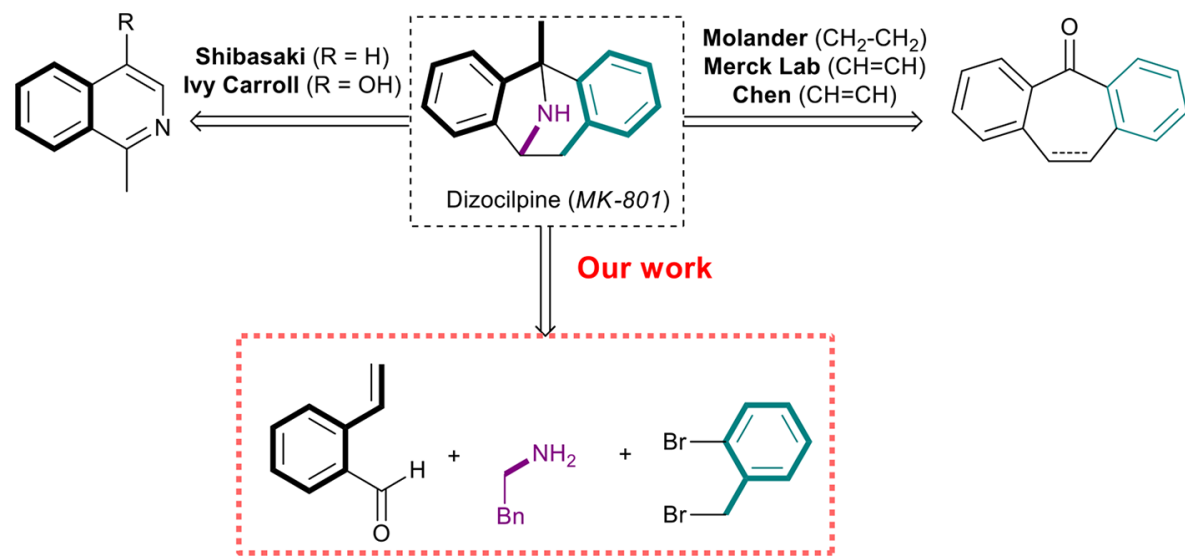

Scheme 2. Retrosynthesis

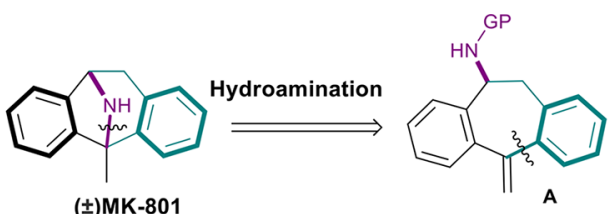

(士)MK-801

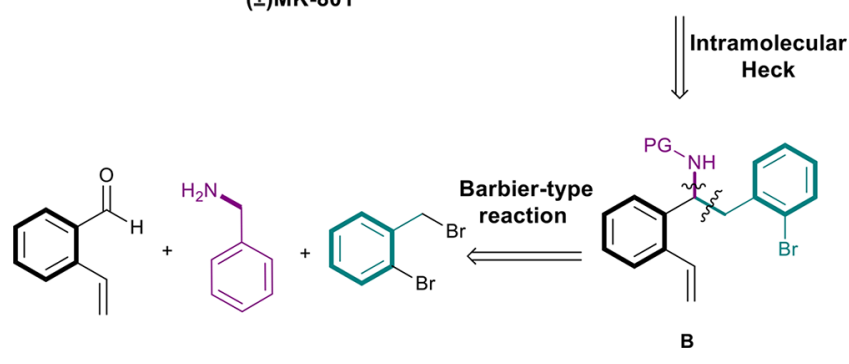

range of substituents, we decided to apply this three component strategy to our building blocks. Wu's methodology only led to 1a with a low yield of $18 \%$ (Table 1, entry 1). Le Gall's method instead provided the desired compound with an improved yield of $41 \%$ (Table 1 , entry 2 ). A small modification of these conditions, i.e., an increase of benzylamine equivalents, pleasingly rose the yield up to $49 \%$.

Table 1. Synthesis of 1a through the Zinc-Promoted BarbierLike Reaction $^{a}$<smiles>C=Cc1ccccc1C=O</smiles>

SM1<smiles>COCCc1ccccc1</smiles><smiles>CCCc1ccccc1Br</smiles>
SM3

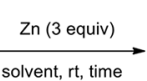<smiles>C=Cc1ccccc1C(Cc1ccccc1Br)NCc1ccccc1</smiles>

1a

\begin{tabular}{cccclcc} 
entry & $\begin{array}{c}\text { SM1 } \\
\text { (equiv) }\end{array}$ & $\begin{array}{c}\text { SM2 } \\
(\text { equiv) }\end{array}$ & $\begin{array}{c}\text { SM3 } \\
\text { (equiv) }\end{array}$ & solvent & $\begin{array}{c}\text { time } \\
(\mathrm{h})\end{array}$ & $\begin{array}{c}\text { yield } \\
(\%)^{b}\end{array}$ \\
\hline 1 & 1 & 2 & 3 & THF & 15 & 18 \\
2 & 1.1 & 1 & 2.5 & MeCN & 1 & 41 \\
3 & 1 & 1.4 & 2.2 & MeCN & 1 & 49
\end{tabular}

${ }^{a}$ Conditions: Under inert atmosphere, a suspension of zinc dust (3 equiv), trifluoroacetic acid ( 0.3 equiv), and benzyl bromide ( 0.4 equiv) in dry acetonitrile was stirred $5 \mathrm{~min}$ at room temperature. Then, benzylamine (1.4 equiv), vinylbenzaldehyde (1 equiv), and 2bromobenzyl bromide ( 2.2 equiv) were added to the solution, and the reaction mixture was stirred at room temperature for $1 \mathrm{~h} .{ }^{b}$ Yield of isolated product.
The next step consists in the formation of a 7-membered ring compound 3a through an intramolecular $\alpha$-Heck reaction. To prevent the formation of an indoline side product during this step, it was necessary to protect the nitrogen atom through a carbamate group ( 2 a obtained in $85 \%$ yield, Table 2 ). This could result from a Buchwald coupling during the palladiumcatalyzed $\mathrm{C}-\mathrm{C}$ bond formation.

Table 2. Synthesis of 7-Membered Ring Compound 3a through an Intramolecular $\alpha$-Heck Reaction ${ }^{a}$

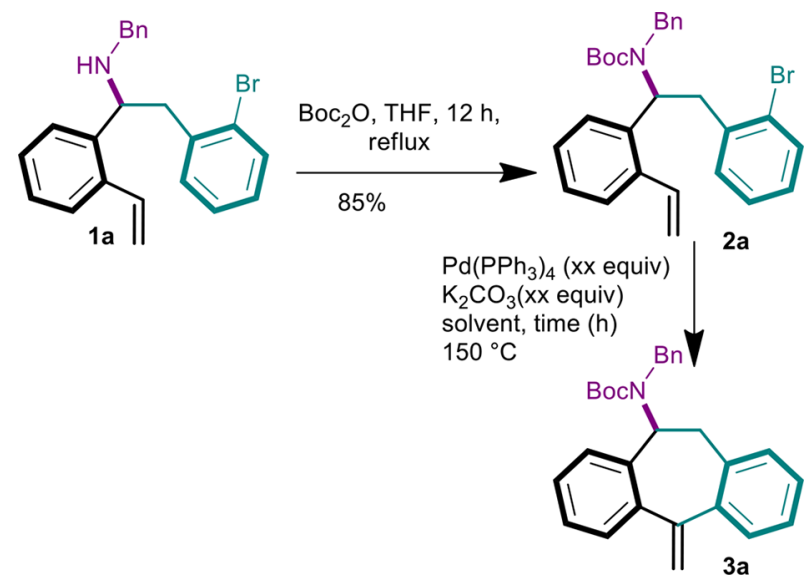

$\begin{array}{cccccc}\text { entry } & \mathrm{Pd}(\mathrm{mol} \%) & \text { base (equiv) } & \text { solvent } & \text { time (h) } & \text { yield }(\%)^{b} \\ 1 & \mathrm{Pd}\left(\mathrm{PPh}_{3}\right)_{4}(50) & 1.2 & \text { MeCN } & 15 & 10^{c} \\ 2 & \mathrm{Pd}\left(\mathrm{PPh}_{3}\right)_{4}(10) & 2 & \text { DMSO } & 30 & 68 \\ 3 & \mathrm{Pd}\left(\mathrm{PPh}_{3}\right)_{4}(20) & 3 & \text { DMSO } & 2 & 90\end{array}$

${ }^{a}$ Under inert atmosphere, to compound $2 \mathrm{a}$ (1 equiv) and $\mathrm{K}_{2} \mathrm{CO}_{3}(x$ equiv) in the solvent was added Pd ( $x$ mol \%), and the mixture was stirred for the suitable time. ${ }^{b}$ Yield of isolated product. ${ }^{c}$ Heated at 85 ${ }^{\circ} \mathrm{C}$.

To favor the formation of the exocyclic alkene, we then optimized the Heck reaction (Table 2). Based on the conditions described by Danishefsky et al. ${ }^{11}$ to generate the exocyclic Taxol alkene, the first catalytic system used consisted of $\mathrm{Pd}\left(\mathrm{PPh}_{3}\right)_{4}\left(0.5\right.$ equiv) in $\mathrm{MeCN}$ at $85{ }^{\circ} \mathrm{C}$. Despite the high catalyst loading ( 0.5 equiv), only $10 \%$ of $3 a$ has been obtained. Changing the solvent from $\mathrm{MeCN}$ to $\mathrm{DMSO}$, in order to carry out the reaction at a higher temperature, resulted in a major improvement with $68 \%$ yield (Table 2 , entry 3 ).With 0.2 equiv of $\mathrm{Pd}\left(\mathrm{PPh}_{3}\right)_{4}$ and 3 equiv of $\mathrm{K}_{2} \mathrm{CO}_{3}$ as the base, the reaction proceeded with an excellent yield of $90 \%$ in only $2 \mathrm{~h}$. 
Table 3. Boc Deprotection of 3a

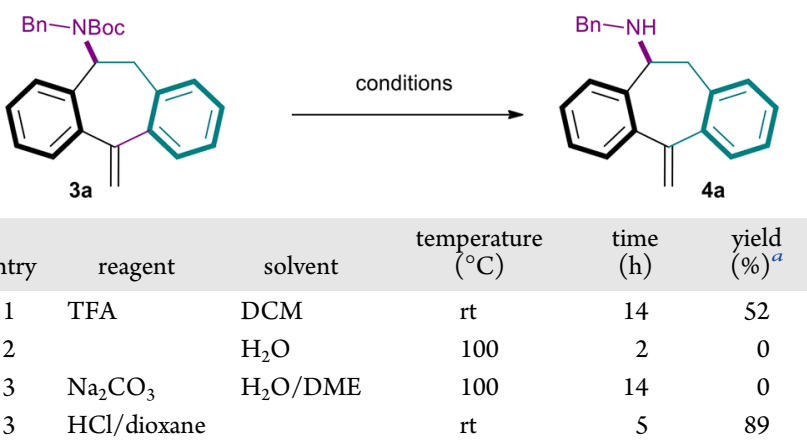

${ }^{a}$ Yield of isolated product.

Scheme 3. $\mathrm{TiCl}_{4}$-Catalyzed Hydroamination
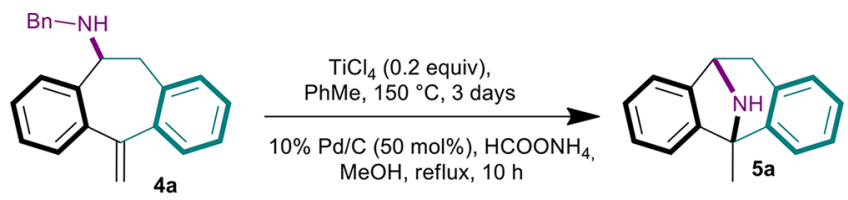

$50 \%$

As reported in Table 3, the deprotection of the amine did not occur as smoothly as we thought in the classical acidic condition, i.e., $20 \%$ (v/v) TFA in DCM. In these conditions, we observed, in a significant amount, a byproduct resulting from the elimination of the benzylamine moiety. We then turned our attention to milder conditions as the one reported by $\mathrm{Qu}$ et al. ${ }^{12}$ Indeed, they described the use of water, at an elevated temperature, as an efficient reagent to remove the Boc protecting group from a large variety of amines. Applying this attractive method to compound 3a was vain since only the starting material was recovered. Treatment of $3 \mathbf{a}$ with sodium carbonate (1.2 equiv) in a mixture of water and DME as depicted by Guillaumet et al. ${ }^{13}$ was not successful in our case. Eventually, using dry $\mathrm{HCl}$ in solution in dioxane followed by a quick basic treatment with potassium carbonate gave $\mathbf{4 a}$ with $89 \%$ yield. $^{14}$

With pure compound $\mathbf{4 a}$ in hand, we then focused on the final cyclization/deprotection reaction sequence. The hydroamination reaction has been widely studied, and a rapid optimization, based on the work of Ackermann et al. ${ }^{15}$ on $\mathrm{TiCl}_{4}$-catalyzed hydroamination of vinylarene, resulted in the bridged protected compound. This intermediate directly, without any purification, underwent a hydrogenation catalyzed by $10 \%$ palladium on carbon with ammonium formate as the hydrogen source. ${ }^{16}$ This reaction required a high catalyst loading ( 0.5 equiv) but afforded the targeted ( \pm )-MK-801 (compound 5a) with 50\% yield over two steps (Scheme 3 ).

To demonstrate the robustness of our strategy, we explored the possibility to extend it to other substrates such as 5-methyl2-vinylbenzaldehyde and 2-bromo-1-(bromomethyl)-4-methoxybenzene. To the best of our knowledge, only a few examples of modified MK-801 are described in the literature. ${ }^{5,17}$ With our methodology, for both substrates, the sequence MCR protection Heck coupling deprotection resulted in the desired compounds $\mathbf{4 b}$ and $\mathbf{4 c}$ with a satisfying yield of, respectively, $46 \%$ and $23 \%$ over 4 steps (Scheme 4 ). In the case of compound $4 \mathrm{~b}$, it appeared that the hydrogenation with Pearlman's catalyst gave a better result than the former method with $\mathrm{HCOONH}_{4}$ and $\mathrm{Pd} / \mathrm{C}$, providing compound $\mathbf{5 b}$ in $51 \%$ yield over 2 steps. For compound 4 c, the sequence previously described afforded compound $5 \mathrm{c}$ in $47 \%$ yield over the two steps.

In conclusion, we successfully developed an original and practical synthesis of dizocilpine and two of its analogues. This method could tolerate more modifications and presents the advantage of requiring simple and commercially available starting materials over the one existing. Efforts are now in progress to synthesis a large library of MK-801 analogues through this strategy to study their biological activity as antagonists of the $N$-methyl-D-aspartate (NMDA) receptor.

\section{EXPERIMENTAL SECTION}

General Information. All reactions were conducted under an argon atmosphere. The solvents, cyclohexane, dichloromethane, 1,4dioxane, ethyl acetate, and methanol, for extraction and chromatography were technical grade. THF was distilled over $\mathrm{Na}$ /benzophenone.

Instrumentation. These compounds were all identified by usual physical methods, e.g., ${ }^{1} \mathrm{H}$ NMR, ${ }^{13} \mathrm{C}$ NMR (J-MOD), IR, and HRMS (ESI). ${ }^{1} \mathrm{H}$ and ${ }^{13} \mathrm{C}$ NMR spectra were measured in $\mathrm{CDCl}_{3}$ with a Bruker Avance-300. ${ }^{1} \mathrm{H}$ chemical shifts are reported in ppm from an internal standard, TMS, or of residual chloroform (7.26 ppm) or methanol (3.32 ppm). The following abbreviations are used: $\mathrm{m}$

\section{Scheme 4. Synthesis of Substituted MK-801 through the Described Strategy}

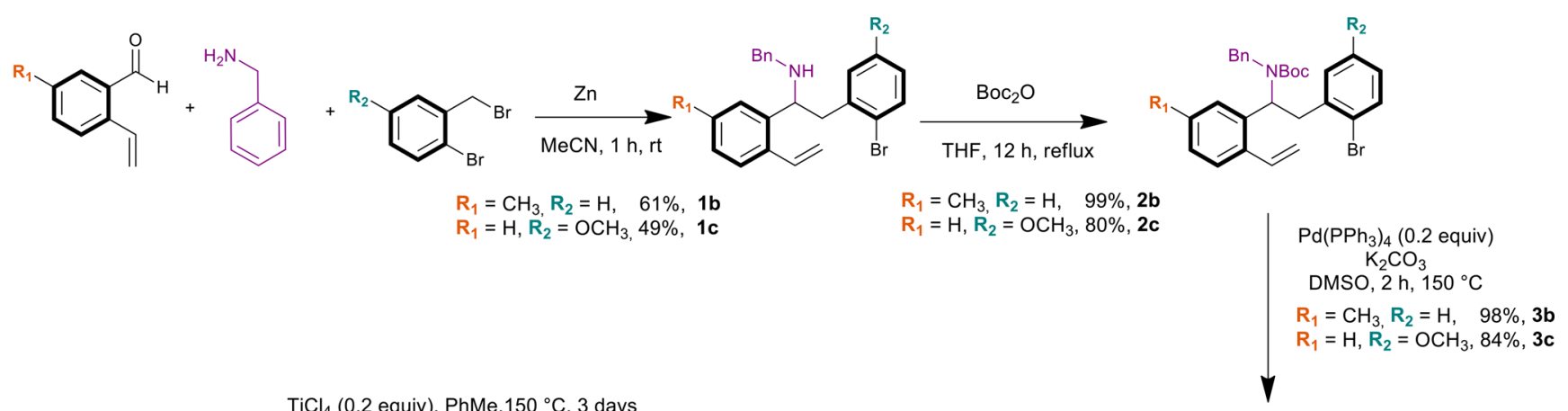

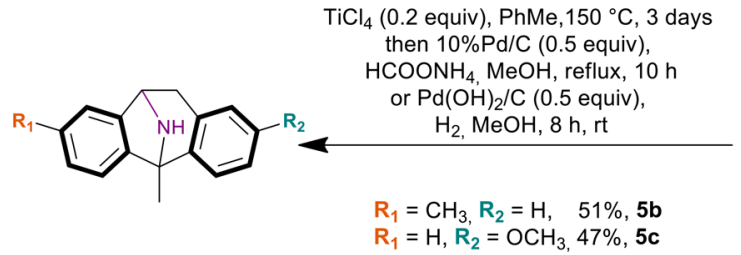
then $10 \% \mathrm{Pd} / \mathrm{C}$ ( 0.5 equiv) $\mathrm{HCOONH}_{4}, \mathrm{MeOH}$, reflux, $10 \mathrm{~h}$ $\mathrm{R}_{1}=\mathrm{H}, \mathrm{R}_{2}=\mathrm{OCH}_{3}, 47 \%, 5 \mathrm{c}$

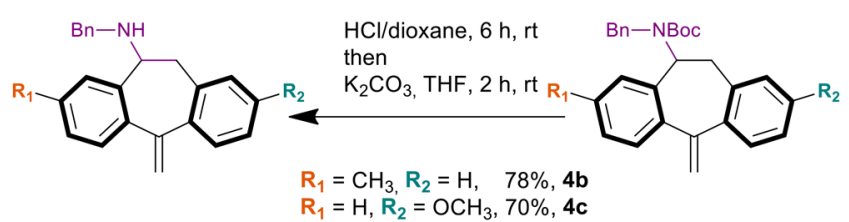

DOI: 10.1021 /acs.joc.8b00305 Org. Chem. 2018, 83, 4264-4269 
(multiplet), s (singlet), $\mathrm{d}$ (doublet), $\mathrm{t}$ (triplet), $\mathrm{dd}$ (doublet of doublets), dt (doublet of triplets), ddd (doublet of doublet of doublets). ${ }^{13} \mathrm{C}$ chemical shifts $(\delta)$ are reported in ppm from a central peak of deuteriochloroform (77.16 ppm) or deuteriomethanol (49.00 $\mathrm{ppm})$. IR spectra were measured on a Bruker Vector 22 spectrophotometer and are reported in wave numbers $\left(\mathrm{cm}^{-1}\right)$. The angles of rotation were measured on a PerkinElmer Polarimeter 341 and denoted as specific rotations, $[\alpha]_{\mathrm{D}}$. High-resolution mass spectra (HRMS) were recorded on a Bruker MicroTOF spectrometer, using ESI with methanol as the carrier solvent. Nominal and exact $\mathrm{m} / \mathrm{z}$ values are reported in daltons. Melting points were recorded on a Büchi B-450 apparatus and are uncorrected. Analytical TLC was performed on Merck precoated silica gel $60 \mathrm{~F}$ plates. Merck silica gel 60 $(0.015-0.040 \mathrm{~mm})$ was used for column chromatography. Flash chromatography was performed on silica gel $60(0.040-0.063 \mathrm{~mm})$ at medium pressure $(200 \mathrm{mbar})$. Compounds were visualized under a UVP Mineralight UVGL-58 lamp $(254 \mathrm{~nm})$ and with vanillin/ $\Delta$ or phosphomolybdic acid $/ \Delta$. Unless otherwise noted, other materials were obtained from commercial suppliers and were used without further purification.

Typical Procedure for the MCR. ${ }^{2}$ A suspension of zinc dust (3 equiv), trifluoroacetic acid ( 0.3 equiv), and benzyl bromide ( 0.4 equiv) in dry acetonitrile was stirred $5 \mathrm{~min}$ at room temperature. Then, benzylamine (1.4 equiv), vinylbenzaldehyde (1 equiv), and 2bromobenzyl bromide ( 2.2 equiv) were added to the solution, and the reaction mixture was stirred at room temperature for $1 \mathrm{~h}$ (exothermic reaction). After the completion of the reaction, the resulting solution was poured into a saturated aqueous $\mathrm{NH}_{4} \mathrm{Cl}$ solution and extracted with EtOAc. The combined organic layers were dried over $\mathrm{Na}_{2} \mathrm{SO}_{4}$ and evaporated to dryness, and the desired compound was purified by column chromatography.

N-Benzyl-2-(2-bromophenyl)-1-(2-vinylphenyl)ethanamine (1a). By following the general procedure for MCR, compound 1a was obtained starting from benzylamine (1.71 g, $16 \mathrm{mmol})$, 2-vinylbenzaldehyde $(1.45 \mathrm{~g}, 11 \mathrm{mmol})$, and 2-bromobenzyl bromide: light yellow oil ( $2.11 \mathrm{~g}$, $5.3 \mathrm{mmol}, 49 \%)$; TLC $R_{f} 0.28$ (c-hexane/EtOAc 90/ 10); IR (neat) $\nu\left(\mathrm{cm}^{-1}\right) 628,660,696,749,773,912,986,1024,1112$, $1158,1205,1361,1440,1469,1495,1567,1625,3026,3062 ;{ }^{1} \mathrm{H}$ NMR $\left(300 \mathrm{MHz}, \mathrm{CDCl}_{3}\right) \delta 7.76(\mathrm{~d}, J=7.7 \mathrm{~Hz}, 1 \mathrm{H}), 7.64-7.54(\mathrm{~m}, 1 \mathrm{H})$, $7.53-6.89(\mathrm{~m}, 12 \mathrm{H}), 5.52(\mathrm{dd}, J=17.3,1.2 \mathrm{~Hz}, 1 \mathrm{H}), 5.24(\mathrm{dd}, J=$ $10.9,1.2 \mathrm{~Hz}, 1 \mathrm{H}), 4.51(\mathrm{t}, J=7.0 \mathrm{~Hz}, 1 \mathrm{H}), 3.77(\mathrm{~d}, J=13.5 \mathrm{~Hz}, 1 \mathrm{H})$, $3.59(\mathrm{~d}, J=13.5 \mathrm{~Hz}, 1 \mathrm{H}), 3.22(\mathrm{dd}, J=13.4,7.8 \mathrm{~Hz}, 1 \mathrm{H}), 3.10(\mathrm{dd}, J=$ 13.4, $6.3 \mathrm{~Hz}, 1 \mathrm{H}), 1.80(\mathrm{~s}, 1 \mathrm{H}) ;{ }^{13} \mathrm{C} \mathrm{NMR}\left(75 \mathrm{MHz}, \mathrm{CDCl}_{3}\right) \delta 140.6$ (C), $140.5(\mathrm{C}), 138.2(\mathrm{C}), 137.8(\mathrm{C}), 134.7(\mathrm{CH}), 132.8(\mathrm{CH}), 131.8$ $(\mathrm{CH}), 128.3(2 \mathrm{CH}), 128.1(2 \mathrm{CH}), 128.0(2 \mathrm{CH}), 127.2(\mathrm{CH}), 127.1$ $(\mathrm{CH}), 126.8(\mathrm{CH}), 126.3(\mathrm{CH}), 125.1(\mathrm{C}), 116.0\left(\mathrm{CH}_{2}\right), 57.2(\mathrm{CH})$, $51.5\left(\mathrm{CH}_{2}\right), 44.5\left(\mathrm{CH}_{2}\right)$; HRMS (ESI-TOF) $m / z[\mathrm{M}+\mathrm{H}]^{+}$calcd for $\mathrm{C}_{23} \mathrm{H}_{22}{ }^{79} \mathrm{BrN}$ 392.1015, found 392.1018 and $\mathrm{m} / z[\mathrm{M}+\mathrm{H}]^{+}$calcd for $\mathrm{C}_{23} \mathrm{H}_{22}{ }^{81} \mathrm{BrN}$ 394.0993, found 394.0995.

$\mathrm{N}$-Benzyl-2-(2-bromophenyl)-1-(5-methyl-2-vinylphenyl)ethanamine $(1 b)$. By following the general procedure for MCR, compound $\mathbf{1 b}$ was obtained starting from benzylamine $(0.4 \mathrm{~mL}, 3.66$ mmol), 5-methyl-2-vinylbenzaldehyde $(297 \mathrm{mg}, 2 \mathrm{mmol})$, and 2bromobenzyl bromide $(1.19 \mathrm{~g}, 4.76 \mathrm{mmol})$ : colorless oil $(512 \mathrm{mg}, 1.26$ mmol, 61\%); TLC $R_{f} 0.2$ (c-hexane/EtOAc 20/1); IR (neat) $\nu\left(\mathrm{cm}^{-1}\right)$ 630, 660, 697, 749, 823, 909, 986, 1025, 1120, 1158, 1199, 1357, 1411, $1439,1469,1566,1609,1734,2922,3027,3062 ;{ }^{1} \mathrm{H}$ NMR (300 MHz, $\left.\mathrm{CDCl}_{3}\right) \delta 7.62(\mathrm{~d}, J=6.6 \mathrm{~Hz}, 1 \mathrm{H}), 7.50-7.00(\mathrm{~m}, 12 \mathrm{H}), 5.53(\mathrm{~d}, J=$ $17.2 \mathrm{~Hz}, 1 \mathrm{H}), 5.22(\mathrm{~d}, J=11.1 \mathrm{~Hz}, 1 \mathrm{H}), 4.60-4.45(\mathrm{~m}, 1 \mathrm{H}), 3.81(\mathrm{~d}, J$ $=13.5 \mathrm{~Hz}, 1 \mathrm{H}), 3.62(\mathrm{~d}, J=13.5 \mathrm{~Hz}, 1 \mathrm{H}), 3.41-3.00(\mathrm{~m}, 2 \mathrm{H}), 2.51(\mathrm{~s}$, $3 \mathrm{H}), 1.83(\mathrm{~s}, 1 \mathrm{H}) ;{ }^{13} \mathrm{C} \mathrm{NMR}\left(75 \mathrm{MHz}, \mathrm{CDCl}_{3}\right) \delta 140.7$ (C), 140.3 (C), $138.2(\mathrm{C}), 137.7(\mathrm{C}), 134.8(\mathrm{C}), 134.4(\mathrm{CH}), 132.8(\mathrm{CH}), 131.8$ (CH), $128.3(2 \mathrm{CH}), 128.1(\mathrm{CH}), 127.9(2 \mathrm{CH}), 127.2(2 \mathrm{CH}), 127.2$ $(\mathrm{CH}), 126.7(\mathrm{CH}), 126.1(\mathrm{CH}), 125.0(\mathrm{C}), 115.1\left(\mathrm{CH}_{2}\right), 57.0(\mathrm{CH})$, $51.5\left(\mathrm{CH}_{2}\right), 44.5\left(\mathrm{CH}_{2}\right), 21.5\left(\mathrm{CH}_{3}\right)$; HRMS (ESI-TOF) $\mathrm{m} / z[\mathrm{M}+$ $\mathrm{H}]^{+}$calcd for $\mathrm{C}_{24} \mathrm{H}_{24}{ }^{79} \mathrm{BrN} 406.1170$, found 406.1173 and $\mathrm{m} / z[\mathrm{M}+$ $\mathrm{H}]^{+}$calcd for $\mathrm{C}_{24} \mathrm{H}_{24}{ }^{81} \mathrm{BrN}$ 408.1150, found 408.1164.

$\mathrm{N}$-Benzyl-2-(2-bromo-5-methoxyphenyl)-1-(2-vinylphenyl)ethanamine (1c). By following the general procedure for MCR, compound $1 \mathrm{c}$ was obtained starting from benzylamine $(1.71 \mathrm{~g}, 16$ mmol), 2-vinylbenzaldehyde (1.45 g, $11 \mathrm{mmol})$, and 1-bromo-2(bromomethyl)-4-methoxybenzene $(7.0 \mathrm{~g}, 25 \mathrm{mmol})$ : colorless oil (2.2 g, 5.39 mmol, 49\%); TLC $R_{f} 0.44$ (c-hexane/EtOAc 80/20); IR (neat) $\nu\left(\mathrm{cm}^{-1}\right) 644,698,733,763,774,801,856,874,912,986$, $1015,1047,1057,1116,1133,1240,1278,1313,1414,1440,1464$, $1478,1572,1595,2834,2933,3026,3061 ;{ }^{1} \mathrm{H}$ NMR $(300 \mathrm{MHz}$, $\left.\mathrm{CDCl}_{3}\right) \delta 7.67-7.62(\mathrm{~m}, 1 \mathrm{H}), 7.42-7.30(\mathrm{~m}, 3 \mathrm{H}), 7.30-7.13(\mathrm{~m}$, $7 \mathrm{H}), 6.95(\mathrm{dd}, J=17.3,10.9 \mathrm{~Hz}, 1 \mathrm{H}), 6.59(\mathrm{dd}, J=8.7,3.1 \mathrm{~Hz}, 1 \mathrm{H})$, $6.40(\mathrm{~d}, J=3.1 \mathrm{~Hz}, 1 \mathrm{H}), 5.41(\mathrm{dd}, J=17.2,1.6 \mathrm{~Hz}, 1 \mathrm{H}), 5.12(\mathrm{dd}, J=$ 11.0, $1.6 \mathrm{~Hz}, 1 \mathrm{H}), 4.39(\mathrm{t}, J=7.1 \mathrm{~Hz}, 1 \mathrm{H}), 3.67(\mathrm{~d}, J=13.3 \mathrm{~Hz}, 1 \mathrm{H})$, $3.55(\mathrm{~d}, J=10.3 \mathrm{~Hz}, 4 \mathrm{H}), 3.10(\mathrm{dd}, J=13.3,7.6 \mathrm{~Hz}, 1 \mathrm{H}), 2.93$ (dd, $J=$ 13.3, $6.5 \mathrm{~Hz}, 1 \mathrm{H}), 1.73(\mathrm{~s}, 1 \mathrm{H}) ;{ }^{13} \mathrm{C} \mathrm{NMR}\left(75 \mathrm{MHz}, \mathrm{CDCl}_{3}\right) \delta 158.6$ (C), 140.6 (C), 140.4 (C), 138.9 (C), 137.9 (C), 134.6 (CH), 133.2 (CH), $128.3(2 \mathrm{CH}), 128.1(\mathrm{CH}), 128.0(2 \mathrm{CH}), 127.1(\mathrm{CH}), 126.8$ $(2 \mathrm{CH}), 126.3(\mathrm{CH}), 116.8(\mathrm{CH}), 116.0\left(\mathrm{CH}_{2}\right), 115.5(\mathrm{C}), 114.4$ $\left(\mathrm{CH}_{2}\right), 57.2(\mathrm{CH}), 55.4\left(\mathrm{CH}_{3}\right), 51.5\left(\mathrm{CH}_{2}\right), 44.7\left(\mathrm{CH}_{2}\right)$; HRMS (ESI-TOF) $m / z[\mathrm{M}+\mathrm{H}]^{+}$calcd for $\mathrm{C}_{24} \mathrm{H}_{24}{ }^{79} \mathrm{BrNO} 422.1120$, found 422.1116 and $m / z[\mathrm{M}+\mathrm{H}]^{+}$calcd for $\mathrm{C}_{24} \mathrm{H}_{24}{ }^{81} \mathrm{BrNO} 424.1099$, found 424.1095 .

Typical Procedure for Boc Protection. A solution of compound 1 ( 1 equiv) and $\mathrm{Boc}_{2} \mathrm{O}$ (1.5 equiv) in THF was stirred at $60{ }^{\circ} \mathrm{C}$ for 12 h. The mixture was then quenched with a saturated $\mathrm{NaHCO}_{3}$ solution and extracted with $\mathrm{CH}_{2} \mathrm{Cl}_{2}$. The combined organic fractions were washed with brine, dried over $\mathrm{Na}_{2} \mathrm{SO}_{4}$, and concentrated under reduced pressure. The crude residue was purified by flash chromatography.

tert-Butyl Benzyl(2-(2-bromophenyl)-1-(2-vinylphenyl)ethyl)carbamate $(2 a)$. By following the general procedure for Boc protection, compound $\mathbf{2 a}$ was obtained starting from compound $\mathbf{1 a}$ (392 mg, $1 \mathrm{mmol}), \mathrm{Boc}_{2} \mathrm{O}(327 \mathrm{mg}, 1.5 \mathrm{mmol})$, and THF $(2 \mathrm{~mL})$ : white solid (418 mg, $0.85 \mathrm{mmol}, 85 \%)$; mp $85-87{ }^{\circ} \mathrm{C}$; TLC $R_{f} 0.71$ (c-hexane/EtOAc 80/20); IR (neat) $\nu\left(\mathrm{cm}^{-1}\right) 630,661,695,731,750$ $781,823,868,914,930,951,964,983,1027,1074,1123,1145,1160$, $1222,1252,1277,1324,1361,1401,1428,1470,1570,1679,2928$, 2969, 3032; ${ }^{1} \mathrm{H}$ NMR $\left(300 \mathrm{MHz}, \mathrm{CDCl}_{3}\right) \delta 7.53(\mathrm{~d}, J=7.9 \mathrm{~Hz}, 1 \mathrm{H})$, 7.50-7.44 (m, 1H), $7.36(\mathrm{~s}, 1 \mathrm{H}), 7.31-7.13(\mathrm{~m}, 5 \mathrm{H}), 7.1-7.05(\mathrm{~m}$, $4 \mathrm{H}), 6.90(\mathrm{br} \mathrm{s}, 2 \mathrm{H}), 5.98(\mathrm{~s}, 1 \mathrm{H}), 5.46(\mathrm{~d}, J=17.1 \mathrm{~Hz}, 1 \mathrm{H}), 5.23(\mathrm{~d}, J$ $=10.2 \mathrm{~Hz}, 1 \mathrm{H}), 4.34(\mathrm{~d}, J=15.5 \mathrm{~Hz}, 1 \mathrm{H}), 4.20(\mathrm{~d}, J=15.4 \mathrm{~Hz}, 1 \mathrm{H})$, $3.33(\mathrm{~d}, J=7.1 \mathrm{~Hz}, 2 \mathrm{H}), 1.22(\mathrm{~s}, 9 \mathrm{H}) ;{ }^{13} \mathrm{C} \mathrm{NMR}\left(75 \mathrm{MHz}, \mathrm{CDCl}_{3}\right) \delta$ 155.3 (C), 139.3 (C), 138.9 (C), 138.2 (C), 136.9 (C), $134.8(\mathrm{CH})$, $132.8(\mathrm{CH}), 131.6(\mathrm{CH}), 128.2(\mathrm{CH}), 127.8(3 \mathrm{CH}), 127.7(2 \mathrm{CH})$, $127.4(1 \mathrm{CH}), 126.6(\mathrm{CH}), 126.5(\mathrm{CH}), 126.3(\mathrm{CH}), 125.5\left(\mathrm{CH}_{2}\right)$, $116.4\left(\mathrm{CH}_{2}\right), 79.8(\mathrm{C}), 54.6(\mathrm{CH}), 38.4\left(\mathrm{CH}_{2}\right), 28.2\left(3 \mathrm{CH}_{3}\right)$; HRMS (ESI-TOF) $m / z[\mathrm{M}+\mathrm{H}]^{+}$calcd for $\mathrm{C}_{28} \mathrm{H}_{30}{ }^{79} \mathrm{BrNO}_{2}$ 492.1538, found 492.1545 and $m / z[\mathrm{M}+\mathrm{H}]^{+}$calcd for $\mathrm{C}_{28} \mathrm{H}_{30}{ }^{81} \mathrm{BrNO}_{2}$ 494.1518, found 494.1536.

tert-Butyl Benzyl(2-(2-bromophenyl)-1-(5-methyl-2-vinylphenyl)ethyl)carbamate (2b). By following the general procedure for Boc protection, compound $\mathbf{2} \mathbf{b}$ was obtained starting from compound $\mathbf{1 b}$ $(510 \mathrm{mg}, 1.25 \mathrm{mmol}), \mathrm{Boc}_{2} \mathrm{O}(360 \mathrm{mg}, 1.65 \mathrm{mmol})$, and THF $(6 \mathrm{~mL})$ : colorless oil $(602 \mathrm{mg}, 1.19 \mathrm{mmol}, 79 \%)$, TLC $R_{f} 0.28$ (c-hexane/ EtOAc 20/1); IR (neat) $\nu\left(\mathrm{cm}^{-1}\right) 662,698,751,825,844,868,910$, $967,1028,1059,1115,1160,1212,1252,1321,1369,1397,1432$, $1455,1474,1495,1688,1755,1811,2932,2982 ;{ }^{1} \mathrm{H}$ NMR $(300 \mathrm{MHz}$, $\left.\mathrm{CDCl}_{3}\right) \delta 7.57(\mathrm{~d}, J=7.5 \mathrm{~Hz}, 1 \mathrm{H}), 7.45-6.84(\mathrm{~m}, 12 \mathrm{H}), 5.95(\mathrm{~s}, 1 \mathrm{H})$, $5.43(\mathrm{~d}, J=16.7 \mathrm{~Hz}, 1 \mathrm{H}), 5.18(\mathrm{~d}, J=13.1 \mathrm{~Hz}, 1 \mathrm{H}), 4.26(\mathrm{dd}, J=41.6$, $26.7 \mathrm{~Hz}, 2 \mathrm{H}), 3.32(\mathrm{~s}, 2 \mathrm{H}), 2.36(\mathrm{~d}, J=36.6 \mathrm{~Hz}, 3 \mathrm{H}), 1.21(\mathrm{~s}, 9 \mathrm{H})$; ${ }^{13} \mathrm{C}$ NMR $\left(75 \mathrm{MHz}, \mathrm{CDCl}_{3}\right) \delta 155.2(\mathrm{C}), 139.3(\mathrm{C}), 138.2(\mathrm{C}), 137.2$ (C), $136.6(\mathrm{C}), 135.9(\mathrm{C}), 134.5(2 \mathrm{CH}), 132.7(2 \mathrm{CH}), 131.6(\mathrm{CH})$, $128.7(2 \mathrm{CH}), 128.1(\mathrm{CH}), 127.6(2 \mathrm{CH}), 127.3(2 \mathrm{CH}), 126.3(2 \mathrm{CH})$, $125.4\left(\mathrm{CH}_{2}\right), 115.4\left(\mathrm{CH}_{2}\right), 79.6(\mathrm{C}), 54.4(\mathrm{CH}), 38.1\left(\mathrm{CH}_{2}\right), 28.1$ $\left(3 \mathrm{CH}_{3}\right), 21.4\left(\mathrm{CH}_{3}\right)$; HRMS (ESI-TOF) $m / z[\mathrm{M}+\mathrm{Na}]^{+}$calcd for $\mathrm{C}_{29} \mathrm{H}_{32} \mathrm{BrNO}_{2} \mathrm{Na} 528.1514$, found 528.1506 .

tert-Butyl Benzyl(2-(2-bromo-5-methoxyphenyl)-1-(2vinylphenyl)ethyl)carbamate (2c). By following the general procedure for Boc protection, compound $2 \mathrm{c}$ was obtained starting from compound 1c (422 mg, $1 \mathrm{mmol}), \mathrm{Boc}_{2} \mathrm{O}(327 \mathrm{mg}, 1.5 \mathrm{mmol})$, and THF $(2 \mathrm{~mL})$ : white solid $(418 \mathrm{mg}, 0.8 \mathrm{mmol}, 80 \%)$; mp $67-69{ }^{\circ} \mathrm{C}$; TLC $R_{f} 0.52$ (c-hexane/EtOAc 80/20); IR (neat) $\nu\left(\mathrm{cm}^{-1}\right) ; 680,696$, 729, 750, 802, 826, 865, 903, 921, 942, 973, 991, 1009, 1023, 1060, 
$1113,1132,1163,1219,1254,1278,1301,1326,1363,1403,1424$, $1456,1474,1572,1599,1673,2834,2930,2969,3010 ;{ }^{1} \mathrm{H}$ NMR $(300$ $\left.\mathrm{MHz}, \mathrm{CDCl}_{3}\right) \delta 7.57-6.90(\mathrm{~m}, 12 \mathrm{H}), 6.80-6.62(\mathrm{~m}, 1 \mathrm{H}), 6.00(\mathrm{~s}$, $1 \mathrm{H}), 5.57(\mathrm{t}, J=28.0 \mathrm{~Hz}, 1 \mathrm{H}), 5.31(\mathrm{t}, J=14.1 \mathrm{~Hz}, 1 \mathrm{H}), 4.31(\mathrm{q}, J=$ $15.5 \mathrm{~Hz}, 2 \mathrm{H}), 3.73(\mathrm{~s}, 3 \mathrm{H}), 3.45-3.16(\mathrm{~m}, 2 \mathrm{H}), 1.29(\mathrm{~s}, 9 \mathrm{H}) ;{ }^{13} \mathrm{C}$ NMR (75 MHz, $\left.\mathrm{CDCl}_{3}\right) \delta 158.8$ (C), 155.3 (C), 139.3 (C), 139.1 (C), $138.8(\mathrm{C}), 136.8(\mathrm{C}), 134.7(\mathrm{CH}), 133.1(\mathrm{CH}), 128.1(2 \mathrm{CH})$, $127.8(2 \mathrm{CH}), 127.6(2 \mathrm{CH}), 127.0(\mathrm{CH}), 126.5(\mathrm{CH}), 126.4(2 \mathrm{CH})$, $116.8(\mathrm{CH}), 116.3\left(\mathrm{CH}_{2}\right), 115.8(\mathrm{C}), 114.3\left(\mathrm{CH}_{2}\right), 79.7(\mathrm{C}), 55.53$ $\left(\mathrm{OCH}_{3}\right), 54.6(\mathrm{CH}), 38.2\left(\mathrm{CH}_{2}\right), 28.1\left(3 \mathrm{CH}_{3}\right) ; \mathrm{HRMS}$ (ESI-TOF) $m / z[\mathrm{M}+\mathrm{Na}]^{+}$calcd for $\mathrm{C}_{29} \mathrm{H}_{32}{ }^{79} \mathrm{BrNO}_{3} \mathrm{Na} 544.1463$, found 544.1467 and $m / z[\mathrm{M}+\mathrm{Na}]^{+}$calcd for $\mathrm{C}_{24} \mathrm{H}_{15}{ }^{81} \mathrm{BrNO}_{3} \mathrm{Na}$ 546.1443, found 546.1457.

Typical Procedure for Heck Coupling. ${ }^{3}$ A sealed tube was equipped with a septum and purged with argon. The tube was charged with compound 2 ( 1 equiv), $\mathrm{K}_{2} \mathrm{CO}_{3}$ ( 3 equiv), $\mathrm{Pd}\left(\mathrm{PPh}_{3}\right)_{4}$ (0.2 equiv), and dry DMSO. The resulting mixture was flushed with argon and was stirred for $2 \mathrm{~h}$ at $150{ }^{\circ} \mathrm{C}$. The resulting slurry was allowed to cool to room temperature, diluted with EtOAc, and washed with a large amount of brine solution. The organic layer was dried over $\mathrm{MgSO}_{4}$, filtered, and concentrated in vacuo. The crude material was purified by flash chromatography.

tert-Butyl Benzyl(5-methylene-10,11-dihydro-5H-dibenzo[a,d][7]annulen-10-yl)carbamate (3a). By following the general procedure for Heck coupling, compound $3 \mathbf{a}$ was obtained starting from compound 2a (492 mg, $1.0 \mathrm{mmol}), \mathrm{K}_{2} \mathrm{CO}_{3}(414 \mathrm{mg}, 3.0 \mathrm{mmol})$, $\mathrm{Pd}\left(\mathrm{PPh}_{3}\right)_{4}(231 \mathrm{mg}, 0.2 \mathrm{mmol})$, and DMSO $(5 \mathrm{~mL})$. Compound 3a was isolated as a mixture of rotamers: white solid $(370 \mathrm{mg}, 0.9 \mathrm{mmol}$, 90\%); mp 127-129 ${ }^{\circ} \mathrm{C}$; TLC $R_{f} 0.41$ (c-hexane/EtOAc 80/20); IR (neat) $\nu\left(\mathrm{cm}^{-1}\right) 663,697,710,740,752,769,784,858,876,902,959$, $1033,1060,1106,1125,1154,1166,1210,1252,1271,1298,1314$, 1364, 1396, 1460, 1485, 1626, 1683, 2933, 2976; ${ }^{1} \mathrm{H}$ NMR (300 MHz, $\left.\mathrm{CDCl}_{3}\right) \delta 7.50-7.07(\mathrm{~m}, 13 \mathrm{H}), 5.91(\mathrm{br} \mathrm{s}, 0.4 \mathrm{H}), 5.67(\mathrm{br} \mathrm{s}, 1 \mathrm{H}), 5.36$ $(\mathrm{m}, 1.2 \mathrm{H}), 4.5(\mathrm{~m}, 0.5 \mathrm{H}), 4.01(\mathrm{~m}, 1 \mathrm{H}), 3.53(\mathrm{~m}, 0.6 \mathrm{H}), 3.1(\mathrm{~m}$, $0.5 \mathrm{H}), 2.75(\mathrm{sl}, 0.4 \mathrm{H}), 1.50(\mathrm{~s}, 9 \mathrm{H}) ;{ }^{13} \mathrm{C} \mathrm{NMR}\left(75 \mathrm{MHz}, \mathrm{CDCl}_{3}\right) \delta$ 156.1 (C), 151.8 (C), 144.2 (C), 140.4 (C), 136.4 (C), 135.5 (C), $135.1(\mathrm{C}), 129.5(\mathrm{CH}), 128.2(\mathrm{CH}), 128.0(\mathrm{CH}), 128.0(\mathrm{CH}), 127.0$ $(2 \mathrm{CH}), 127.0(2 \mathrm{CH}), 126.7(2 \mathrm{CH}), 126.4(\mathrm{CH}), 117.0\left(\mathrm{CH}_{2}\right), 80.3$ (C), $58.4(\mathrm{CH}), 38.2\left(\mathrm{CH}_{2}\right), 37.6\left(\mathrm{CH}_{2}\right), 28.6\left(3 \mathrm{CH}_{3}\right)$; HRMS (ESITOF) $m / z[\mathrm{M}+\mathrm{Na}]^{+}$calcd for $\mathrm{C}_{28} \mathrm{H}_{29} \mathrm{NO}_{2} \mathrm{Na} 434.2096$, found 434.2089.

tert-Butyl Benzyl(8-methyl-5-methylene-10,11-dihydro-5Hdibenzo[a,d][7]annulen-10-yl)carbamate (3b). By following the general procedure for Heck coupling, compound $\mathbf{3 b}$ was obtained starting from compound $\mathbf{2 b}(192 \mathrm{mg}, 0.38 \mathrm{mmol}), \mathrm{K}_{2} \mathrm{CO}_{3}(173.5 \mathrm{mg}$, $1.25 \mathrm{mmol}), \mathrm{Pd}\left(\mathrm{PPh}_{3}\right)_{4}(45 \mathrm{mg}, 0.03 \mathrm{mmol})$, and DMSO $(0.9 \mathrm{~mL})$. Compound $\mathbf{3 b}$ was isolated as a mixture of rotamers: colorless oil (160 $\mathrm{mg}, 0.37 \mathrm{mmol}, 98 \%)$, TLC R 0.42 (c-hexane/EtOAc 20/1); ${ }^{1} \mathrm{H}$ NMR $\left(300 \mathrm{MHz}, \mathrm{CDCl}_{3}\right) \delta 7.43-6.84(\mathrm{~m}, 13 \mathrm{H}), 5.84(\mathrm{br} \mathrm{s}, 0.5 \mathrm{H}), 5.62(\mathrm{br}$ $\mathrm{s}, 0.5 \mathrm{H}), 5.28(\mathrm{~m}, 1 \mathrm{H}), 4.36(\mathrm{~m}, 0.6 \mathrm{H}), 3.92(\mathrm{br} \mathrm{s}, 1 \mathrm{H}), 3.5(\mathrm{~m}, 0.7 \mathrm{H})$, $3.11(\mathrm{~m}, 0.5 \mathrm{H}), 2.2(\mathrm{~s}, 3 \mathrm{H}), 1.50(\mathrm{~s}, 9 \mathrm{H}) ;{ }^{13} \mathrm{C}$ NMR $(75 \mathrm{MHz}$, $\left.\mathrm{CDCl}_{3}\right) \delta 156.4$ (C), 151.7 (C), 144.5 (C), 140.3 (C), 137.8 (C), $135.7(\mathrm{C}), 135.0(\mathrm{C}), 129.6(\mathrm{CH}), 129.4(\mathrm{CH}), 128.6(\mathrm{CH}), 128.0$ $(2 \mathrm{CH}), 127.0(2 \mathrm{CH}), 126.6(2 \mathrm{CH}), 116.5\left(\mathrm{CH}_{2}\right), 80.2(\mathrm{C}), 58.4$ $(\mathrm{CH}), 48.6\left(\mathrm{CH}_{2}\right), 38.1\left(\mathrm{CH}_{2}\right), 37.3\left(\mathrm{CH}_{2}\right), 28.6\left(3 \mathrm{CH}_{3}\right)$; IR (neat) $\nu$ $\left(\mathrm{cm}^{-1}\right)$ 1042, 1053, 1078, 1228, 1253, 1381, 1407, 1452, 1964, 2208, 2340, 2362, 2902, 2970, 2988; HRMS (ESI-TOF) $\mathrm{m} / z[\mathrm{M}+\mathrm{Na}]^{+}$ calcd for $\mathrm{C}_{29} \mathrm{H}_{31} \mathrm{NO}_{2} \mathrm{Na} 448.2252$, found 448.2254 .

tert-Butyl Benzyl(2-methoxy-5-methylene-10,11-dihydro-5Hdibenzo[a,d][7]annulen-10-yl)carbamate (3c). By following the general procedure for Heck coupling, compound $3 \mathrm{c}$ was obtained starting from compound $2 \mathrm{c}(522 \mathrm{mg}, 1 \mathrm{mmol}), \mathrm{K}_{2} \mathrm{CO}_{3}(414 \mathrm{mg}, 3$ $\mathrm{mmol}), \mathrm{Pd}\left(\mathrm{PPh}_{3}\right)_{4}(231 \mathrm{mg}, 0.2 \mathrm{mmol})$, and DMSO $(5 \mathrm{~mL})$. Compound 3a was isolated as a mixture of rotamers: white solid ( 371 $\mathrm{mg}, 0.8 \mathrm{mmol}, 84 \%)$; mp $46-48{ }^{\circ} \mathrm{C}$; TLC $R_{f} 0.26$ (c-hexane/EtOAc 90/10); IR (neat) $\nu\left(\mathrm{cm}^{-1}\right) 621,643,706,741,774,792,815,858$, $872,894,910,935,963,1028,1077,1090,1116,1157,1261,1281$, $1295,1314,1332,1365,1390,1424,1447,1496,1608,1684,2839$, 2973; ${ }^{1} \mathrm{H}$ NMR $\left(300 \mathrm{MHz}, \mathrm{CDCl}_{3}\right) \delta 7.32-6.94(\mathrm{~m}, 12 \mathrm{H}), 6.75-6.55$ $(\mathrm{m}, 1.7 \mathrm{H}), 6.4(\mathrm{br} \mathrm{s}, 0.4 \mathrm{H}), 5.65(\mathrm{br} \mathrm{s}, 0.4 \mathrm{H}), 5.48(\mathrm{~s}, 1 \mathrm{H}), 5.15(\mathrm{~s}$,
$1 \mathrm{H}), 4.32(\mathrm{~m}, 0.5 \mathrm{H}), 3.94(\mathrm{~m}, 1 \mathrm{H}), 3.94-3.58(\mathrm{~m}, 4 \mathrm{H}), 3.37(\mathrm{~m}$, $0.7 \mathrm{H}), 2.93(\mathrm{~m}, 0.5 \mathrm{H}), 1.34$ (br s, $9 \mathrm{H}) ;{ }^{13} \mathrm{C} \mathrm{NMR}\left(75 \mathrm{MHz}, \mathrm{CDCl}_{3}\right) \delta$ 159.5 (C), 156.3 (C), 151.3 (C), 140.3 (C), 136.7 (C), 136.3 (C), $131.1(\mathrm{CH}), 129.4(2 \mathrm{CH}), 128.6(\mathrm{CH}), 128.0(2 \mathrm{CH}), 127.8(\mathrm{CH})$, $127.0(2 \mathrm{CH}), 127.0(\mathrm{CH}), 126.5(\mathrm{CH}), 116.7\left(\mathrm{CH}_{2}\right), 114.2(\mathrm{CH})$, $112.4(\mathrm{CH}), 111.8(\mathrm{CH}), 80.2(\mathrm{C}), 58.4(\mathrm{CH}), 48.6(\mathrm{CH}), 38.5$ $\left(\mathrm{CH}_{2}\right), 37.9\left(\mathrm{CH}_{2}\right), 28.5\left(3 \mathrm{CH}_{3}\right)$; HRMS (ESI-TOF) $m / z[\mathrm{M}+\mathrm{H}]^{+}$ calcd for $\mathrm{C}_{29} \mathrm{H}_{32} \mathrm{NO}_{3}$ 442.2382, found 442.2381.

Typical Procedure for the Amine Deprotection. ${ }^{4}$ Under an inert atmosphere, at $0{ }^{\circ} \mathrm{C}, \mathrm{HCl}$ (80 equiv, $4 \mathrm{M}$ in dioxane) was added to compound 3 ( 1 equiv). The mixture was then stirred at room temperature for $5 \mathrm{~h}$. The solvent was removed by rotary evaporation, and the crude reaction was used directly for the next step without any purification. Dry THF and $\mathrm{K}_{2} \mathrm{CO}_{3}$ (2 equiv) were added to the residue, and the stirring was maintained for $2 \mathrm{~h}$ at room temperature. The resulting suspension was filtered through a Celite pad and rinsed with EtOAc. The filtrate was concentrated in vacuo, and the crude material was purified by silica gel chromatography.

$\mathrm{N}$-Benzyl-5-methylene-10,11-dihydro-5H-dibenzo[a,d][7]annulen-10-amine (4a). By following the general procedure for the amine deprotection, compound $\mathbf{4 a}$ was obtained starting from compound 3a (411 mg, $1.0 \mathrm{mmol})$ : white solid $(277 \mathrm{mg}, 0.89$ mmol, 89\%); mp 264-266 ${ }^{\circ} \mathrm{C}$; TLC R 0.25 (c-hexane/EtOAc 90/10); IR (neat) $\nu\left(\mathrm{cm}^{-1}\right)$ 697, 727, 753, 779, 904, 975, 1028, 1037, 1073, $1093,1118,1158,1202,1254,1269,1328,1452,1486,1601,1616$, $1668,1695,1723,2920,3026,3062 ;{ }^{1} \mathrm{H}$ NMR $\left(300 \mathrm{MHz}, \mathrm{CDCl}_{3}\right) \delta$ 7.44-7.33 (m, 4H), 7.37-7.13 (m, 9H), 5.45 (dd, $J=14.3,1.6 \mathrm{~Hz}$, $2 \mathrm{H}), 4.25(\mathrm{dd}, J=7.4,3.2 \mathrm{~Hz}, 1 \mathrm{H}), 3.76(\mathrm{~s}, 2 \mathrm{H}), 3.41-3.26(\mathrm{~m}, 2 \mathrm{H})$; ${ }^{13} \mathrm{C}$ NMR $\left(75 \mathrm{MHz}, \mathrm{CDCl}_{3}\right.$ ) $\delta 151.8$ (C), 140.7 (C), $140.2(\mathrm{C}), 139.8$ (C), $135.1(2 \mathrm{C}), 130.6(\mathrm{CH}), 128.5(2 \mathrm{CH}), 128.4(\mathrm{CH}), 128.4(\mathrm{CH})$, $128.3(\mathrm{CH}), 127.9(\mathrm{CH}), 127.9(\mathrm{CH}), 127.2(\mathrm{CH}), 127.1(\mathrm{CH})$, $126.6(\mathrm{CH}), 117.4\left(\mathrm{CH}_{2}\right), 58.5(\mathrm{CH}), 51.4\left(\mathrm{CH}_{2}\right), 39.2\left(\mathrm{CH}_{2}\right)$; HRMS (ESI-TOF) $m / z[\mathrm{M}+\mathrm{H}]^{+}$calcd for $\mathrm{C}_{23} \mathrm{H}_{21} \mathrm{~N} 312.1728$, found 312.1723

$\mathrm{N}$-Benzyl-8-methyl-5-methylene-10,11-dihydro-5H-dibenzo[a,d][7]annulen-10-amine (4b). By following the general procedure for the amine deprotection, compound $\mathbf{4 b}$ was obtained starting from compound $3 \mathbf{b}(300 \mathrm{mg}, 0.7 \mathrm{mmol})$ : yellowish oil (180 mg, 0.55 mmol, 78\%); TLC $R_{f} 0.17$ (c-hexane/EtOAc 80/20); IR (neat) $\nu$ $\left(\mathrm{cm}^{-1}\right) 804,880,1045,1088,1274,1326,1380,1408,1453,2361$, 2901, 2974; ${ }^{1} \mathrm{H}$ NMR (300 MHz, $\left.\mathrm{CDCl}_{3}\right) \delta 7.45-7.07(\mathrm{~m}, 12 \mathrm{H}), 5.46$ $(\mathrm{d}, J=9.1 \mathrm{~Hz}, 2 \mathrm{H}), 4.23(\mathrm{dd}, J=7.3,3.1 \mathrm{~Hz}, 1 \mathrm{H}), 3.84(\mathrm{~s}, 2 \mathrm{H}), 3.44-$ $3.28(\mathrm{~m}, 2 \mathrm{H}), 2.41(\mathrm{~s}, 3 \mathrm{H}), 1.79(\mathrm{~s}, 1 \mathrm{H}) ;{ }^{13} \mathrm{C}$ NMR $\left(75 \mathrm{MHz}, \mathrm{CDCl}_{3}\right)$ $\delta 151.6(\mathrm{C}), 140.6$ (C), 140.4 (C), 139.9 (C), 137.7 (C), 137.6 (C), $135.1(\mathrm{C}), 130.5(\mathrm{CH}), 128.7(\mathrm{CH}), 128.4(2 \mathrm{CH}), 128.4(2 \mathrm{CH})$, $128.2(\mathrm{CH}), 127.8(\mathrm{CH}), 127.7(\mathrm{CH}), 127.0(\mathrm{CH}), 126.5(\mathrm{CH})$, 117.1 $\left(\mathrm{CH}_{2}\right), 58.6(\mathrm{CH}), 51.5\left(\mathrm{CH}_{2}\right), 39.2\left(\mathrm{CH}_{2}\right), 21.0\left(\mathrm{CH}_{3}\right)$; HRMS (ESI-TOF) $\mathrm{m} / z[\mathrm{M}+\mathrm{H}]^{+}$calcd for $\mathrm{C}_{24} \mathrm{H}_{23} \mathrm{~N} 326.1909$, found 326.1918

N-Benzyl-2-methoxy-5-methylene-10,11-dihydro-5H-dibenzo$[a, d][7] a n n u l e n-10$-amine (4c). By following the general procedure for the amine deprotection, compound $4 \mathrm{c}$ was obtained starting from compound $3 \mathrm{c}(441.6 \mathrm{mg}, 1.0 \mathrm{mmol})$ : white solid $(239 \mathrm{mg}, 0.7 \mathrm{mmol}$, $70 \%$ ); mp 264-266 ${ }^{\circ} \mathrm{C}$; TLC $R_{f} 0.35$ (c-hexane/EtOAc 80/20); IR (neat) $\nu\left(\mathrm{cm}^{-1}\right) 634,650,667,697,717,731,750,761,783,801,824$, $847,865,881,902,919,944,973,944,973,1016,1041,1068,1081$, $1101,1124,1231,1270,1306,1323,1343,1375,1409,1434,1460$, 1499, 1604, 1650, 1698, 2327, 2341, 2361, 2902, 2989; ${ }^{1} \mathrm{H}$ NMR (300 $\left.\mathrm{MHz}, \mathrm{CDCl}_{3}\right) \delta 7.39-7.18(\mathrm{~m}, 10 \mathrm{H}), 6.73(\mathrm{dd}, J=8.5,2.7 \mathrm{~Hz}, 1 \mathrm{H})$, $6.67(\mathrm{~d}, J=2.7 \mathrm{~Hz}, 1 \mathrm{H}), 5.39(\mathrm{dd}, J=26.2,1.8 \mathrm{~Hz}, 2 \mathrm{H}), 4.21(\mathrm{dd}, J=$ 7.5, $3.4 \mathrm{~Hz}, 1 \mathrm{H}), 3.76(\mathrm{~s}, 5 \mathrm{H}), 3.36-3.18(\mathrm{~m}, 2 \mathrm{H}), 1.83(\mathrm{~s}, 1 \mathrm{H}) ;{ }^{13} \mathrm{C}$ NMR $\left(75 \mathrm{MHz}, \mathrm{CDCl}_{3}\right) \delta 159.3(\mathrm{C}), 151.2(\mathrm{C}), 141.2(\mathrm{C}), 140.6$ (C), 140.0 (C), $136.6(\mathrm{C}), 132.4(\mathrm{C}), 129.6(\mathrm{CH}), 128.5(2 \mathrm{CH})$, $128.4(2 \mathrm{CH}), 128.2(\mathrm{CH}), 127.8(\mathrm{CH}), 127.4(\mathrm{CH}), 127.2(\mathrm{CH})$, $127.0(\mathrm{CH}), 116.5\left(\mathrm{CH}_{2}\right), 115.7(\mathrm{C}), 112.2(\mathrm{CH}), 58.6(\mathrm{CH}), 55.4$ $(\mathrm{CH})$, $51.6\left(\mathrm{CH}_{3}\right), 40.1\left(\mathrm{CH}_{2}\right)$; HRMS (ESI-TOF) $m / z[\mathrm{M}+\mathrm{H}]^{+}$ calcd for $\mathrm{C}_{24} \mathrm{H}_{23} \mathrm{NO} 342.1858$, found 342.1865.

Typical Procedure for the Cyclization/Hydrogenation. ${ }^{5}$ A sealed tube was charged with dry toluene, compound 4 ( 1 equiv), and $\mathrm{TiCl}_{4}$ ( 0.2 equiv). The tube was flushed with argon, and the resulting 
mixture was stirred for 3 days at $150{ }^{\circ} \mathrm{C}$. After the completion of the reaction, the solvent was evaporated under reduced pressure. The crude reaction was used directly for the next step without any purification. Anhydrous ammonium formate (15 equiv), 10\% Pd/C ( 0.5 equiv), and dry methanol were added in a single portion under nitrogen. The resulting reaction mixture was stirred at reflux temperature. After the completion of the reaction, the catalyst was removed by filtration through a Celite pad and rinsed with chloroform. The filtrate was evaporated under reduced pressure, and the resulting crude material was purified by flash chromatography.

5-Methyl-10, 11-dihydro-5H-5, 10-epiminodibenzo[a,d][7]annulene (5a). By following the general procedure for cyclization/ hydrogenation, compound 5a was obtained starting from compound 4 a $(312 \mathrm{mg}, 1.0 \mathrm{mmol})$ : colorless oil ( $110 \mathrm{mg}, 0.50 \mathrm{mmol}, 50 \%)$; TLC $R_{f} 0.24$ (MeOH/DCM 5/95); IR (neat) $\nu\left(\mathrm{cm}^{-1}\right)$ 631, 691, 713, 725, 741, 754, 772, 799, 844, 1014, 1032, 1048, 1061, 1095, 1117, 1202, $1244,1296,1344,1375,1423,1458,1488,1731,2928,2961,3065 ;{ }^{1} \mathrm{H}$ NMR $\left(300 \mathrm{MHz}, \mathrm{CDCl}_{3}\right) \delta 7.31-7.24(\mathrm{~m}, 2 \mathrm{H}), 7.14-7.03(\mathrm{~m}, 5 \mathrm{H})$, 6.96-6.92 (m, 1H), $4.70(\mathrm{~d}, J=5.6 \mathrm{~Hz}, 1 \mathrm{H}), 3.45(\mathrm{dd}, J=16.8,5.7$ $\mathrm{Hz}, 1 \mathrm{H}), 2.73(\mathrm{~d}, J=16.8 \mathrm{~Hz}, 1 \mathrm{H}), 2.54(\mathrm{~s}, 1 \mathrm{H}), 1.92(\mathrm{~s}, 3 \mathrm{H}) ;{ }^{13} \mathrm{C}$ NMR (75 MHz, $\mathrm{CDCl}_{3}$ ) $\delta 152.2$ (C), 144.7 (C), 144.4 (C), 132.4 (C), $130.3(\mathrm{CH}), 127.2(\mathrm{CH}), 127.1(\mathrm{CH}), 126.7(\mathrm{CH}), 125.8(\mathrm{CH})$, $121.7(\mathrm{CH}), 121.5(\mathrm{CH}), 118.7(\mathrm{CH}), 64.3(\mathrm{C}), 58.5(\mathrm{CH}), 34.6$ $\left(\mathrm{CH}_{2}\right), 20.2\left(\mathrm{CH}_{3}\right)$; HRMS (ESI-TOF) $\mathrm{m} / z[\mathrm{M}+\mathrm{H}]^{+}$calcd for $\mathrm{C}_{16} \mathrm{H}_{15} \mathrm{~N}$ 222.1283, found 222.1275.

2-Methoxy-5-methyl-10,11-dihydro-5H-5,10-epiminodibenzo$[a, d][7] a n n u l e n e(5 \mathrm{c})$. By following the general procedure for cyclization/hydrogenation, compound $\mathbf{5 c}$ was obtained starting from compound $4 \mathrm{c}(341 \mathrm{mg}, 1.0 \mathrm{mmol})$ : colorless oil $(118 \mathrm{mg}, 0.47 \mathrm{mmol}$, 47\%); TLC $R_{f} 0.17\left(\mathrm{MeOH} / \mathrm{DCM} \mathrm{3/97);} \mathrm{IR} \mathrm{(neat)} \nu\left(\mathrm{cm}^{-1}\right) 651\right.$, 695, 732, 760, 799, 817, 842, 895, 980, 1030, 1069, 1143, 1193, 1253, $1273,1311,1342,1377,1407,1455,1496,1576,1606,2360,2901$, 2970, 2988; ${ }^{1} \mathrm{H}$ NMR $\left(300 \mathrm{MHz}, \mathrm{CDCl}_{3}\right) \delta 7.43-6.96(\mathrm{~m}, 6 \mathrm{H}), 6.61$ $(\mathrm{d}, J=3 \mathrm{~Hz}, 1 \mathrm{H}), 6.49(\mathrm{~s}, 1 \mathrm{H}), 4.87(\mathrm{~s}, 1 \mathrm{H}), 4.71(\mathrm{~d}, J=2.1 \mathrm{~Hz}, 1 \mathrm{H})$, $3.68(\mathrm{~s}, 3 \mathrm{H}), 3.45(\mathrm{dd}, J=16.8,5.0 \mathrm{~Hz}, 1 \mathrm{H}), 2.72(\mathrm{~d}, J=17.1 \mathrm{~Hz}, 1 \mathrm{H})$, $1.91(\mathrm{~s}, 3 \mathrm{H}) ;{ }^{13} \mathrm{C}$ NMR $\left(75 \mathrm{MHz}, \mathrm{CDCl}_{3}\right) \delta 158.9,151.7,143.6$, 136.2, 133.5, 127.3, 127.1, 126.8, 122.6, 121.8, 118.5, 115.5, 111.2, 64.1, 58.2, 55.2, 34.6, 19.9; HRMS (ESI-TOF) $\mathrm{m} / z[\mathrm{M}+\mathrm{H}]^{+}$calcd for $\mathrm{C}_{17} \mathrm{H}_{17} \mathrm{NO} 252.1388$, found 252.1385 .

5,8-Dimethyl-10,11-dihydro-5H-5,10-epiminodibenzo[a,d][7]annulene $(5 b)$. In a sealed tube, under argon, to a solution of $N$ benzyl-8-methyl-5-methylene-10,11-dihydro-5H-dibenzo[a,d][7]annulen-10-amine $(144 \mathrm{mg}, 0.44 \mathrm{mmol})$ in dry toluene $(1 \mathrm{~mL})$ was added $\mathrm{TiCl}_{4}(0.09 \mathrm{~mL}, 0.09 \mathrm{mmol}, 1 \mathrm{M}$ in DCM $)$. The resulting mixture was stirred for 3 days at $150{ }^{\circ} \mathrm{C}$. After the completion of the reaction, the solvent was evaporated under reduced pressure. The crude reaction was used directly for the next step without any purification. A total of $10 \mathrm{~mL}$ of $\sim 15 \% \mathrm{Pd}(\mathrm{OH})_{2} / \mathrm{C}(200 \mathrm{mg}, 0.16$ mmol, $50 \%$ in $\mathrm{H}_{2} \mathrm{O}$ ) was added to the crude material solubilized in dry methanol $(4 \mathrm{~mL})$. The mixture was stirred under a hydrogen atmosphere for $8 \mathrm{~h}$. After the completion of the reaction, the catalyst was removed by filtration through a Celite pad, which was then rinsed with DCM. The filtrate was evaporated to dryness and purified by flash chromatography to afford the desired product 5,8-dimethyl-10,11dihydro-5H-5,10-epiminodibenzo[ $a, d][7]$ annulene as a yellowish oil (40.4 mg, $0.17 \mathrm{mmol}, 51 \%$ ): TLC $R_{f} 0.31$ (DCM/MeOH 95:5); IR (neat) $\nu\left(\mathrm{cm}^{-1}\right) 630,665,711,742,773,810,836,853,880,904,929$, 966, 989, 1034, 1070, 1103, 1117, 1229, 1266, 1293, 1321, 1341, 1375, 1425, 1451, 1484, 1615, 1713, 2854, 2921, 2968; ${ }^{1} \mathrm{H}$ NMR $(300 \mathrm{MHz}$, $\left.\mathrm{CDCl}_{3}\right) \delta 7.18-7.14(\mathrm{~m}, 1 \mathrm{H}), 7.04-6.97(\mathrm{~m}, 3 \mathrm{H}), 6.88-6.83(\mathrm{~m}$, $3 \mathrm{H}), 4.62(\mathrm{~d}, J=5.6 \mathrm{~Hz}, 1 \mathrm{H}), 3.54-3.35(\mathrm{~m}, 3 \mathrm{H}), 2.65(\mathrm{~d}, J=16.9$ $\mathrm{Hz}, 1 \mathrm{H}), 2.21(\mathrm{~s}, 3 \mathrm{H}), 1.84(\mathrm{~s}, 3 \mathrm{H}) ;{ }^{13} \mathrm{C} \mathrm{NMR}\left(75 \mathrm{MHz}, \mathrm{CDCl}_{3}\right) \delta$ 148.9 (C), 144.2 (C), 144.0 (C), 136.7 (C), 132.1 (C), $130.3(\mathrm{CH})$, $127.8(\mathrm{CH}), 125.9(\mathrm{CH}), 122.6(\mathrm{CH}), 121.5(\mathrm{CH}), 118.5(\mathrm{CH}), 64.3$ (C), $58.4\left(\mathrm{CH}_{3}\right), 34.4\left(\mathrm{CH}_{2}\right), 21.4(\mathrm{CH}), 20.0\left(\mathrm{CH}_{3}\right)$; HRMS (ESITOF) $m / z[\mathrm{M}+\mathrm{H}]^{+}$calcd for $\mathrm{C}_{17} \mathrm{H}_{17} \mathrm{~N} 236.1439$, found 236.1436 .

\section{ASSOCIATED CONTENT}

\section{S Supporting Information}

The Supporting Information is available free of charge on the ACS Publications website at DOI: 10.1021/acs.joc.8b00305.

Spectroscopic data of all compounds (PDF)

\section{AUTHOR INFORMATION}

\section{Corresponding Authors}

*E-mail: samir.messaoudi@u-psud.fr.

*E-mail: mouad.alami@u-psud.fr.

ORCID

Samir Messaoudi: 0000-0002-4994-9001

Author Contributions

${ }^{\#}$ K.H.V.R. and E.Y.-P. contributed equally.

Notes

The authors declare no competing financial interest.

\section{ACKNOWLEDGMENTS}

Authors acknowledge support of this project by CNRS, University Paris Sud and by La Ligue Contre le Cancer through an Equipe Labellisée 2014 grant. This work was funded by LabEx LERMIT (Laboratory of Excellence in Research on Medication and Innovative Therapeutics) under the "Investment for the Future" program ANR-11-IDEX-0003-01 within the ANR-10-LABX-0033 (to S.C.-K. and M.A.) and Agence Nationale de la Recherche (ANR) "Biomedical Innovation" program 2014(DS0404) within ANR-14-CE16-0016 (to S.C.K.)

\section{REFERENCES}

(1) Wong, E. H.; Kemp, J. A.; Priestley, T.; Knight, A. R.; Woodruff, G. N.; Iversen, L. L. Proc. Natl. Acad. Sci. U. S. A. 1986, 83, 71047108.

(2) Funabashi, K.; Ratni, H.; Kanai, M.; Shibasaki, M. J. Am. Chem. Soc. 2001, 123, 10784-10785.

(3) Constable, K. P.; Blough, B. E.; Carroll, F. I. Chem. Commun. 1996, 717-718.

(4) Molander, G. A.; Dowdy, E. D. J. Org. Chem. 1999, 64, 65156517.

(5) Thompson, W. J.; Anderson, P. S.; Britcher, S. F.; Lyle, T. a.; Thies, J. E.; Magill, C. a.; Varga, S. L.; Schwering, J. E.; Lyle, P. a.; Christy, M. E. J. Med. Chem. 1990, 33, 789-808.

(6) Chang, M. Y.; Huang, Y. P.; Lee, T. W.; Chen, Y. L. Tetrahedron 2012, 68, 3283-3287.

(7) Vautravers, N. R.; Regent, D. D.; Breit, B. Chem. Commun. 2011, 47, 6635-6637.

(8) Domling, A. Chem. Rev. 2006, 106, 17-89.

(9) Fan, R.; Pu, D.; Qin, L.; Wen, F.; Yao, G.; Wu, J. J. Org. Chem. 2007, 72, 3149-3151.

(10) Le Gall, E.; Haurena, C.; Sengmany, S.; Martens, T.; Troupel, M. J. Org. Chem. 2009, 74, 7970-9973.

(11) Young, W. B.; Masters, J. J.; Danishefsky, S. J. Am. Chem. Soc. 1995, 117, 5228-5234.

(12) Wang, J.; Liang, Y.-L.; Qu, J. Chem. Commun. 2009, 14, 51445146.

(13) El Kazzouli, S.; Koubachi, J.; Berteina-Raboin, S.; Mouaddib, A.; Guillaumet, G. Tetrahedron Lett. 2006, 47, 8575-8578.

(14) Han, G.; Tamaki, M.; Hruby, V. J. J. Pept. Res. 2001, 58, 338341.

(15) Kaspar, L. T.; Fingerhut, B.; Ackermann, L. Angew. Chem., Int. Ed. 2005, 44, 5972-5974.

(16) Ram, S.; Spicer, L. Tetrahedron Lett. 1987, 28, 515-516.

(17) Schultz, D. M.; Wolfe, J. P. Org. Lett. 2011, 13, 2962-2965. 